\title{
Diffuse idiopathic pulmonary neuroendocrine cell hyperplasia (DIPNECH)
}

\begin{abstract}
Introduction: Diffuse idiopathic pulmonary neuroendocrine cells hyperplasia (DIPNECH) is an extremely rare lesion of the lung, less than 400 cases are reported in the literature since its discovery in 1992.

Observation: 51-year-old Patient hospitalized after the fortuitous discovery of a small nodule of the lobe apical right. The surgical pathologic examination of the scannoguided biopsy found a lung parenchyme the bronchial cells of which are replaced by neuroendocrine hyperplasiques making cells posing the diagnosis of a Diffuse idiopathic pulmonary neuroendocrine cell hyperplasia with a tumor typical carcinoïde. The patient benefited a surgical resection of the nodule. The contrôle by a thoracic computed tomography scans don't objectivited a parenchymal recurrence, or appearance of other nodule.
\end{abstract}

Conclusion: The current data of this disease concerning its diagnosis, treatment and prognosis are still little known. Several studies will be necessary to clear up the various persistent points about this pathology.

Keywords: DIPNECH; lung; neuroendocrine cell hyperplasia
Volume 5 Issue 4 - 2018

\begin{abstract}
Mohamed Sinaa,' Mohamed Oukabli, Abderrahmane Albouzidi²

'Department of pathology, military hospital Moulay Ismail, Morocco

${ }^{2}$ Department of pathology, military hospital MohammedV, Morocco
\end{abstract}

Correspondence: Mohamed Sinaa, department of pathology, Moulay Ismail military hospital , Meknes, Morocco, Email sinaamohamed57@gmail.com

Received: July 09, 2018 | Published: July 23, 2018

\section{Introduction}

Diffuse idiopathic pulmonary neuroendocrine cells hyperplasia (diffuse idiopathic pulmonary neuroendocrine cell hyperplasia: DIPNECH) is a rare pulmonary lesion described and named since 1992. ${ }^{1}$ It is characterized by a proliferation of neuroendocrine cells in the bronchial mucosa not exceeding the basal membrane. It may be associated with typical carcinoid tumors. ${ }^{2}$ The WHO classification of pulmonary tumors classifies it among pre-neoplastic lung lesions, with an increased risk of carcinoid tumors. ${ }^{2}$ We report here a case of a patient with pulmonary neuroendocrine hyperplasia, poorly known because of its low prevalence and non-specific presentation.

\section{Case report}

A 51-year-old woman, clinically asymptomatic who hospitalized following the discovery by a systematic radiography of a nodule of the right apical lobe. The clinical examination was normal. A thoracic computed tomography (CT) scans was performed which confirmed the presence of a small nodule of $12 \times 16 \mathrm{~mm}$ (Figure 1). Bronchic fibroscopy showed a diffuse inflammatory of the bronchial tree without a tumoral bud. A scannoguided biopsy was performed. The microscopic study revealed a pulmonary parenchyma whose bronchial cells are replaced by neuroendocrine cells carrying appareance of diffuse idiopathic hyperplasia of the neuroendocrine cells (Figure 2), associated with a tumor proliferation made of small nests (Figure 3 ) of cells with a eosinophilic cytoplasm, and a homogeneous nuclei with a molted chromatin, without mitoses (Figure 4). The stroma is vascular endocrinoid. The immunohistochemical complement showed a positivity of the tumor cells described to synaptophysin (Figure 5) and to chromogranin (Figure 6). The final diagnosis is diffuse idiopathic pulmonary neuroendocrine cells hyperplasia (DIPNECH), associated with a typical carcinoid tumor. The patient was benefecied of resection of this nodule. Three years after the operation, the patient is still alive and no tumor recurrence has been detected so far.

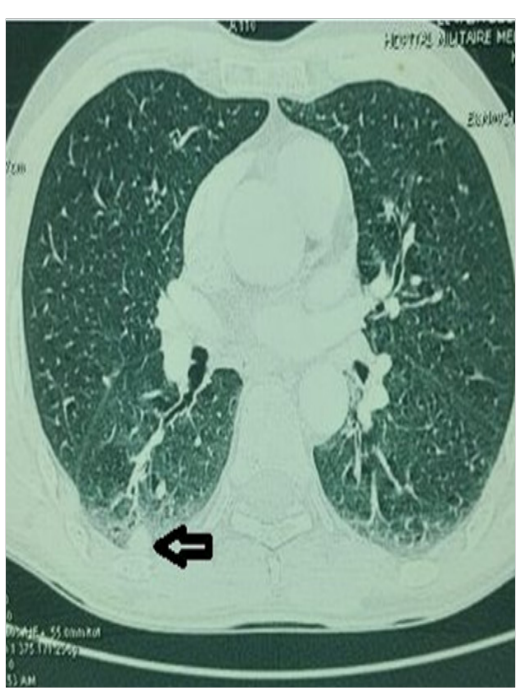

Figure I Thoracic CT scan showing the presence of an apical lobe nodule of the right lung (arrow).

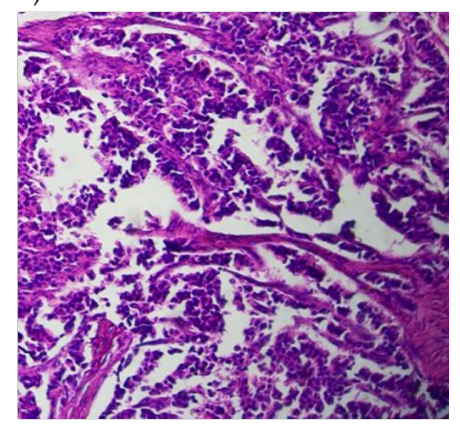

Figure 2 Pulmonary parenchyma whose bronchial cells are replaced by neuroendocrine cells carrying out aspects of diffuse idiopathic hyperplasia of neuroendocrine cells (HEx200). 


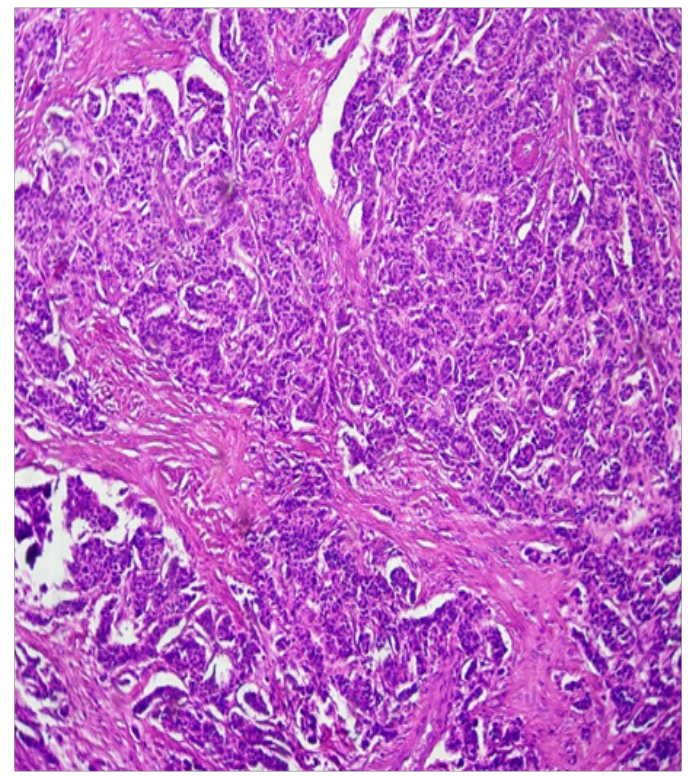

Figure 3 Neuroendocrine tumor proliferation made of small nests (HExI00).

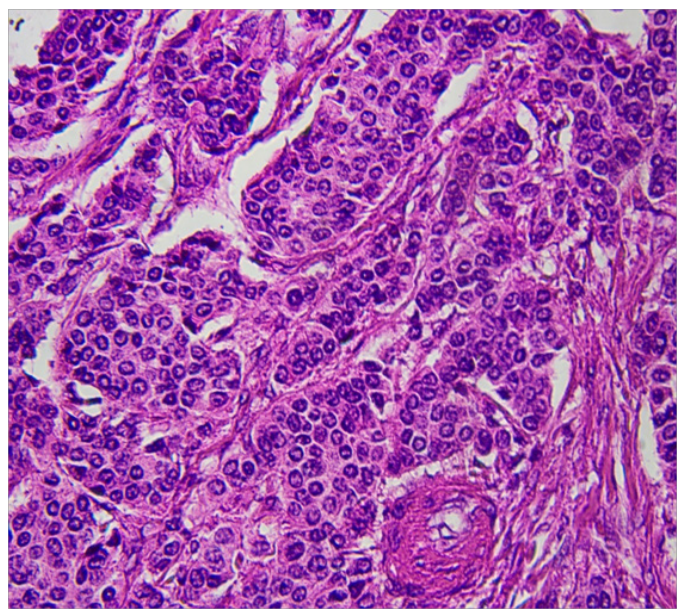

Figure 4 Tumor cells have an eosinophilic cytoplasm, and have homogeneous nuclei, without mitoses (HEx200).

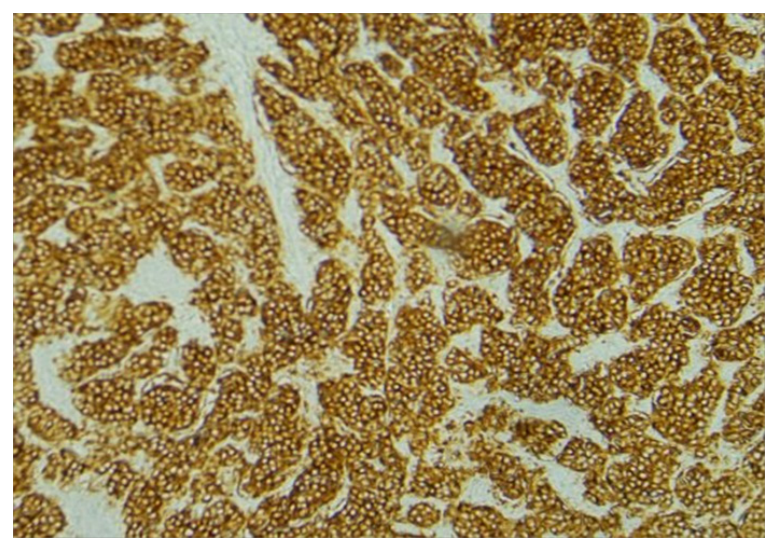

Figure 5 Positivity of tumor cells to synaptophysin (x200).

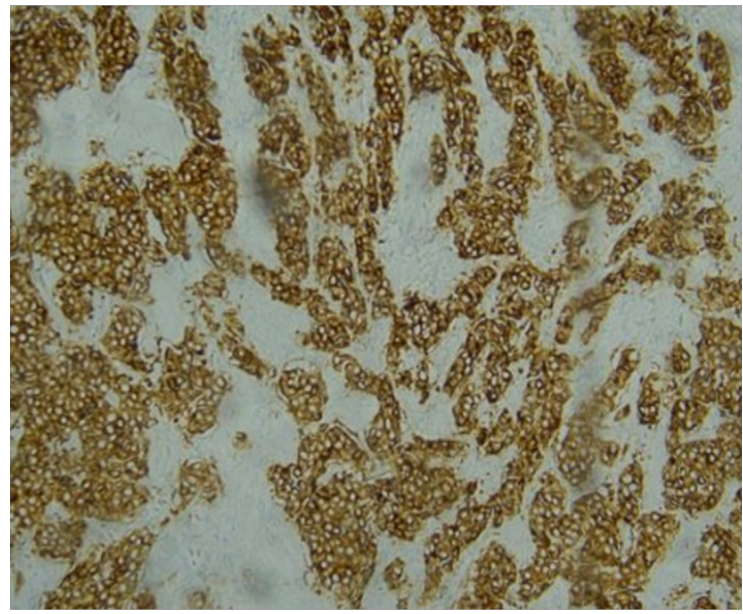

Figure 6 Positivity of tumor cells to chromogranin (x200).

\section{Discussion}

Diffuse idiopathic pulmonary neuroendocrine cells hyperplasia (DIPNECH) is an extremely rare pulmonary lesion, fewer than 400 cases have been reported in the literature since its discovery in 1992. 80\% of the cases described are observed in Europe and North America. About $10 \%$ in Africa and $10 \%$ in Asia and South America. ${ }^{2,3}$ The low prevalence of DIPNECH allows it to be studied only in case report or in small series. ${ }^{3,4}$ This pulmonary disease is characterized by a proliferation of pulmonary neuroendocrine cells disseminated in the bronchial wall, in contact with the basal membrane of bronchiolar epithelium, and by the frequent but inconstant association of neuroendocrine tumors. ${ }^{4}$ DIPNECH observed, according to the cases reported in the literature, in the great majority of women (sex ratio to 8/1) and preferentially non-smokers, unlike our patient, with a median age near 50 Years. ${ }^{4,5}$ The pathophysiology of this preneoplastic lesion is unknown until now.

Clinical symptomatology is not specific. There seem to be as many symptomatic patients with nonspecific signs of chronic obstructive pulmonary disease as in asymptomatic patients as in our patient. The functional respiratory profile of these patients is mainly found in an obstructive ventilatory disorder, generally moderate, which may be related to neuroendocrine infiltration of the bronches and bronchioles. ${ }^{6}$ The discovery of this pathology is often the result of an incidental radiological examination or a number of cases in the context of the monitoring of anterior cancer treated. The CT scan typically reveals the presence of single or multiple nodules. Bronchial dilation and thickening of the bronchial wall may be associated. The CT scan may still be normal. ${ }^{7,8}$

DIPNECH is not generally exceeding $1 \mathrm{~cm}$ to $1.5 \mathrm{~cm}$ in size. Microscopically, it is characterized by a proliferation of neuroendocrine cells which replaces the bronchial epithelium in the form of small nests. These cells have an eosinophilic cytoplasm, homogeneous nuclei, nucleolated, a chromatin with the appearance of "pepper and salt", without cytonular atypia, or mitotic figures. These cells are positive for neuroendocrine markers: synaptophysin and chromogranin. The association with typical carcinoid tumors is inconstant. ${ }^{6-8}$ The therapeutic management of this entity remains 
difficult to codify. Bronchial semeiology could be improved by corticosteroid therapy. The main problem is the attitude to be adopted with regard to multiple pulmonary nodular lesions. The reference treatment, currently proposed, associate a pulmonary resection or lobectomy with mediastinal lymph node dissection. An isotopic treatment may be considered, but the small size of the lesions can lead to absence of fixation by the octreoscan scintigraphy. ${ }^{8,9}$

The progression is slow with a rather favorable prognosis and a 5-year survival of $91 \% .{ }^{9}$ Clinical, computed tomography and endoscopic monitoring therefore seems acceptable, aiming at detecting a recurrence requiring a large surgical excision. Slow progress probably permits annual monitoring, which should be extended indefinitely.

\section{Conclusion}

We report a case of DIPNECH, a well defined entity with an atypical clinical and paraclinic presentation. Only the pathological examination allows a diagnosis of certainty. The classical microscopic aspect is a diffuse proliferation of neuroendocrine cells within the bronchiolar epithelium. Slow progression allows extended clinical, computed tomography and endoscopic monitoring. Surgical excision is the reference treatment.

\section{Acknowledgments}

None.

\section{Conflict of interest}

The authors declare that they have no conflicts of interests.

\section{References}

1. Foran PJ, Hayes SA, Blair DJ, et al. Imaging appearances of diffuse idiopathic pulmonary neuroendocrine cell hyperplasia. Clin Imaging.
2015;39(2):243-246

2. Nassar AA, Jaroszewski DE, Helmers RA, et al. Diffuse idiopathic pulmonary neuroendocrine cell hyperplasia: a systematic overview. Am J Respir Crit Care Med. 2011;184(1):8-16.

3. Gorshtein A, Gross DJ, Barak D, et al. Diffuse idiopathic pulmonary neuroendocrine cell hyperplasia and the associated lung neuroendocrine tumors: clinical experience with a rare entity. Cancer. 2012;118(3):612-619.

4. Lee JS, Brown KK, Cool C, et al. Diffuse pulmonary neuroendocrine cell hyperplasia: radiologic and clinical features. $J$ Comput Assist Tomogr. 2002;26(2):180-184.

5. Wirtschafter E, Walts A, Liu ST, et al. Diffuse Idiopathic Pulmonary Neuroendocrine Cell Hyperplasia of the Lung (DIPNECH): Current Best Evidence. Lung. 2015;193(5):659-667.

6. Warth A, Herpel E, Schmähl A, et al, Diffuse idiopathic pulmonary neuroendocrine cell hyperplasia (DIPNECH) in association with an adenocarcinoma: a case report. Journal of Medical Case Reports. 2008;2:21.

7. Baniak NM, Wilde B, Kanthan R. Diffuse idiopathic pulmonary neuroendocrine cell hyperplasia (DIPNECH)--An uncommon precursor of a common cancer? Pathol Res Pract. 2016;212(2):125-129.

8. Gosney JR, Williams IJ, Dodson AR, et al. Morphology and antigen expression profile of pulmonary neuroendocrine cells in reactive proliferations and diffuse idiopathic pulmonary neuroendocrine cell hyperplasia (DIPNECH). Histopathology. 2011;59(4):751-762.

9. Marchevsky AM, Walts AE. Diffuse idiopathic pulmonary neuroendocrine cell hyperplasia (DIPNECH). Semin Diagn Pathol. 2015;32(6):438-444. 